\title{
Invariant natural killer $\mathrm{T}$ cells as sensors and managers of inflammation
}

\author{
Luc Van Kaer, Vrajesh V. Parekh, and Lan Wu \\ Department of Pathology, Microbiology and Immunology, Vanderbilt University School of \\ Medicine, Nashville, TN 37232, USA
}

\begin{abstract}
Invariant natural killer T (iNKT) cells are a subset of innate-like lymphocytes that recognize glycolipid antigens bound by the major histocompatibility complex (MHC)-class-I-related protein CD1d. iNKT cells are activated early during a variety of infections and inflamma-tory diseases and contribute to the subsequent development of adaptive immune responses. Consequently, iNKT cells play a critical role in the development and resolution of inflammatory diseases and represent attractive targets for the development of immunotherapies. Recent studies have provided important insight into the mechanisms by which iNKT cells become activated in response to diverse inflammatory stimuli. These new findings should be instrumental to promote the immunomodulatory properties of iNKT cells for treatment of inflammatory diseases.
\end{abstract}

\section{Keywords}

invariant natural killer T cells; CD1d; glycolipids; inflammation; immunotherapy

\section{Phenotype and effector functions of iNKT cells}

NKT cells are a group of T lymphocytes that share surface markers with cells of the NK cell lineage (for reviews on NKT cells, see [1-5]). Distinct subsets of NKT cells and related cell types have been identified (Box 1). Most NKT cells, referred to as Type I or invariant NKT cells, express a rearranged $\mathrm{T}$ cell receptor (TCR) with a semi-invariant TCRa chain (Va14$\mathrm{Ja} 18$ in mice and the homologous Va24-Ja18 in humans) and a restricted set of TCR $\beta$ chains. The TCR of iNKT cells recognizes lipid or glycolipid antigens bound by the MHCclass-I-related protein CD1d, which is expressed by antigen-presenting cells (APCs) and some other cell types such as hepatocytes. Although a few early studies suggested that some CD1d-restricted T cells could also react with peptide antigens, this idea has largely been abandoned, although it was recently revived in a study suggesting that some murine NKT cells react with a collagen-derived peptide [6]. iNKT cells also express the NK cell marker CD161 (called NK1.1 in mice) as well as NK cell inhibitory receptors such as members of the murine Ly-49 receptor family. Most iNKT cells express CD4 and constitutively express multiple activation markers such as CD25, CD69, and CD122, and these cells, at least in mice, constitutively express mRNAs for several cytokines. iNKT cells are most abundant in liver; represent a substantial lymphocyte subset in thymus, spleen, peripheral blood, bone marrow, and fat tissue; and can also be detected in lymph nodes, lung and the gastrointestinal mucosa. iNKT cells in the lung and liver are predominantly associated with the vasculature $[7,8]$, and in the spleen these cells are located within the marginal zone and

(C) 2012 Elsevier Ltd. All rights reserved.

Corresponding author: Van Kaer, L. (luc.van.kaer@vanderbilt.edu).. 
red pulp [8], placing these cells in a strategic location for rapid sensing of airborne or bloodborne antigens. The prevalence and effector functions of iNKT cells vary among mouse strains. Compared with mice, humans have fewer iNKT cells and exhibit wide variations in the number of circulating iNKT cells.

Consistent with their activated phenotype, engagement of the TCR on iNKT cells results in the rapid secretion of a variety of cytokines, including $\mathrm{T}$ helper (Th) 1 cytokines such as interferon (IFN)- $\gamma$ and tumor necrosis factor (TNF)- $a$ and Th2 cytokines such as interleukin (IL)-4 and IL-13, and acquisition of cytotoxic activity [1-5]. More recent studies have shown that a subset of CD4 ${ }^{+}$NK1.1 ${ }^{-}$iNKT cells can produce the Th17 cytokines IL-17 and IL-22 [9,10], iNKT cells can produce the follicular helper T cell cytokine IL-21 [11-13], and transforming growth factor (TGF)- $\beta$ can convert iNKT cells into suppressor cells expressing the forkhead box 3 (Foxp3) transcription factor [14,15]. In contrast with classical $\mathrm{T}$ cells from the adaptive immune system, iNKT cells are unable to develop immunological memory. Instead, following in vivo activation by potent glycolipid antigens or microbial molecular patterns, these cells typically become resistant to restimulation [16]. The innate-like effector functions of iNKT cells are imparted during the intrathymic development of these cells, in response to induction of the transcription factor promyelocytic leukemia zinc finger (PLZF) $[17,18]$.

Activated iNKT cells can interact with a variety of other cell types, including NK cells, dendritic cells (DCs), macrophages, and neutrophils of the innate immune system and classical B and T lymphocytes of the adaptive immune system [1-5]. As such, iNKT cells can participate in a variety of immune responses, ranging from infections to cancer and autoimmune and inflammatory diseases. However, until recently, the mechanisms by which iNKT cells become activated during a variety of diseases have remained elusive. Here, we discuss the molecular mechanisms that permit iNKT cells to respond to a diverse array of inflammatory stimuli and review recent progress in the capacity of these cells to modulate inflammatory responses and diseases.

\section{Mechanisms of iNKT cell activation during inflammation}

iNKT cells become activated during a variety of infections and inflammatory conditions [15]. Although some infectious agents and other exogenous stimuli contain lipid antigens that are recognized by iNKT cells, most microorganisms lack cognate iNKT cell antigens and instead activate iNKT cells in a manner that is driven by cytokines in conjunction with endogenous antigens. Similar mechanisms might be at play during the activation of iNKT cells in various inflammatory conditions, although additional mechanisms that can lead to iNKT cell activation have been identified.

\section{TCR-mediated iNKT cell activation by exogenous lipid antigens}

Several microorganisms that contain cognate lipid antigens for iNKT cells have been identified [19] (Figure 1a). These antigens include: a-glucuronosylceramides from Sphingomonas species; diacylglycerol-containing glycolipids from Borrelia burgdorferi and Streptococcus species (including Streptococcus pneumoniae and Group B streptococci) [20]; a cholesteryl a-glucoside from Helicobacter pylori [21]; tetramannosylated phosphatidylinositol $\left(\mathrm{PIM}_{4}\right.$ ) from Mycobacterium bovis; and lipophosphoglycan from Leishmania donovani. Although the antigens from Sphingomonas, B. burgdorferi, Streptococcus and Helicobacter appear to be recognized by most (if not all) mouse and human iNKT cells, those derived from $M$. bovis and $L$. donovani are only recognized by a small fraction of iNKT cells. Additional studies have suggested that some human iNKT cells can react with phospholipids derived from grass pollen [22], and that house dust, a major 
cause of airway inflammation, contains iNKT cell stimuli, including glycosphingolipids and other antigens that are most likely of bacterial origin [23].

The Sphingomonas antigens bear significant structural resemblance to a-galactosylceramide (a-GalCer) from the sea sponge Agelas mauritianus, which was the first glycolipid antigen shown to be recognized by the majority of both mouse and human iNKT cells [24] (Box 2). Sphingomonas species are ubiquitous in the environment, including soil and the ocean. Although unproven, it is likely that a-GalCer was derived from Sphingomonas bacteria that colonized the marine sponges.

Synthetic versions of these exogenous iNKT cell antigens have been employed extensively to investigate the glycolipid antigen specificity of iNKT cells, structural aspects of the interactions between the invariant TCR with CD1d-bound glycolipid antigens, and the immunomodulatory activities of glycolipid-activated iNKT cells. These studies have shown that the invariant TCR functions as a pattern-recognition receptor that can bind with lipid antigens containing diverse structures [25]. Although most studies have focused on a-linked glycolipids, which are present in microbes but not mammals, it is now clear that iNKT cells can react with some $\beta$-linked glycolipids as well, and this includes self-antigens such as $\beta$ glycosylceramides ( $\beta$-GlcCers) [26-28]. Cognate lipid antigens typically can induce both IFN- $\gamma$ and IL-4 secretion by iNKT cells. Studies with these synthetic glycolipids have provided a wealth of information regarding the immunomodulatory properties of iNKT cells, which has resulted in significant interest in their use as vaccine adjuvants and immunotherapeutics (Box 2).

\section{Cytokine-driven iNKT cell activation}

Most microorganisms that can activate iNKT cells in vivo lack cognate iNKT cell antigens. In fact, one study has suggested that, even for those microorganisms that contain iNKT cell antigens, the main mechanism of iNKT cell activation is driven by cytokines [29]. This pathway of iNKT cell activation was first described for Salmonella typhimurium [30]. APCs exposed to this microorganism were able to induce IFN- $\gamma$ production by iNKT cells.

Exposure of APCs to bacterial products such as Salmonella lipopolysaccharide (LPS) is similarly capable of inducing IFN- $\gamma$ production in iNKT cells, therefore, it is clear that this type of iNKT cell activation is independent of CD1d-presented microbial lipid antigens. Antibodies against IL-12 and CD1d significantly inhibit iNKT cell activation in these cultures. These findings suggest a pathway of iNKT cell activation involving induction of proinflammatory cytokines such as IL-12 by APCs in response to activation by Toll-like receptor (TLR) agonists, together with signaling through the invariant TCR in response to engagement with CD1d-presented self antigens (Figure 1b). This indirect pathway of iNKT cell activation is now widely accepted as a general mechanism of iNKT cell stimulation in the absence of exogenous lipid antigens (reviewed in [19]). iNKT cells activated in this manner produce IFN- $\gamma$ but usually not IL- 4 . Additional studies have shown that a variety of TLR agonists, including agonists of TLR2, TLR4, TLR5, TLR8, and TLR9 can activate iNKT cells either in vitro or in vivo [31-34]. This indirect iNKT cell activation pathway is not restricted to microbial patterns that engage TLRs. iNKT cell activation by several fungal microorganisms involves signaling through the $\beta$-glucan receptor Dectin- 1 on APCs [35]. Thus, it is conceivable that recognition of diverse danger signals, including microbial, dietary and endogenous, damage-associated molecular patterns by APCs can stimulate iNKT cells. For most microbes or their products, secretion of IL-12 by APCs is critical, but in some cases, other proinflammatory cytokines appear to play a more important role. Type I IFNs play a dominant role in iNKT cell activation by the TLR9 agonist CpG [33], and IL-18 is more critical than IL-12 in the response of iNKT cells to Escherichia coli LPS, a TLR4 agonist [36]. Most studies have also confirmed the requirement for weak TCR-CD1d 
interactions in iNKT cell activation by microbial products, although CD1d expression is not always required, as seen in the response of iNKT cells to APCs stimulated with E. coli LPS [36] and infection by murine cytomegalovirus [32], and CD1d expression plays a minor role in the iNKT cell response to CpG-treated APCs [31-33].

Some microbes and microbial products can induce CD1d expression on APCs, in a manner that is dependent on cytokines such as IFN- $\beta$ or TNF- $\alpha$ [19]. An increase in CD1d expression levels on APCs has been shown to enhance iNKT cell activation [37]. Therefore, increased CD1d expression suggests a potential mechanism for the synergy between cytokine- and TCR-mediated signals in iNKT cell activation. However, the majority of microbes and microbial products fail to induce CD1d expression and several microorganisms instead interfere with CD1d expression or CD1d-restricted antigen presentation [19]. Nevertheless, it is possible that induction of CD1d expression on APCs can play a role during iNKT cell activation in response to infection. Induction of CD1d expression during inflammatory conditions may similarly contribute to iNKT cell activation in a variety of disease conditions.

The requirement for TCR-mediated signaling in the response of iNKT cells to most microbes or their products has lead to the notion that these stimuli can induce the production of endogenous lipid antigens for iNKT cells. It is well established that infection and inflammation can induce alterations in the expression of enzymes that control lipid metabolism and thus influence lipid expression [38]. Several studies have shown that activation of iNKT cells by certain microbial products is suppressed by inhibitors of glycosphingolipid synthesis, and that the expression of several enzymes involved in the synthesis of glycosphingolipids is altered in the activated APCs [33,34,39-41]. The idea that glycosphingolipids might serve as self antigens for iNKT cells stems from earlier work, demonstrating that inhibitors of glycosphingolipid synthesis can curb the autoreactivity of murine iNKT cell hybridomas, and that cells deficient in $\beta$-glucosylceramide ( $\beta$-GluCer) synthase are unable to stimulate autoreactive iNKT cells [42]. This proposed role of glycosphingolipids as iNKT cell self antigens is also consistent with the finding that the autoreactivity of murine iNKT cells requires CD1d to access endosomal or lysosomal compartments [43], where glycosphingolipids are present. Subsequent studies have identified the $\beta$-linked glycosphingolipid isoglobotrihexosylceramide (iGb3) as a potential self antigen able to activate both murine and human iNKT cells, albeit weakly [44], and additional studies suggest a role for iGb3 or related glycosphingolipids with terminal Nacetyl-galactosamine sugars as self antigens involved in iNKT cell activation following microbial products $[41,44]$. However, the role of iGb3 as a self antigen for iNKT cells is unclear, because it has proven difficult to detect iGb3 in most tissues and cell types $[45,46]$, and because iGb3 synthase-deficient mice have normal iNKT cell numbers and functions [47]. Furthermore, iGb3 is unlikely to be important for self-reactivity of human iNKT cells, because humans lack a functional iGb3 synthase and, unlike mouse iNKT cells, autoreactivity of many human iNKT cells does not require recycling of CD1d molecules to endosomal or lysosomal compartments [48]. A recent study has further suggested that globosides rather than isoglobosides are endogenous ligands that mediate iNKT cell autoreactivity [49]. Although most studies focus on glycosphingolipids as self antigens, there is also evidence that other types of antigens can contribute to the autoreactivity of murine iNKT cells [50]. Some human iNKT cells can react with phospholipids [51] and many human iNKT cells react with lysophosphatidylcholine, which is induced during inflammation [52], but these antigens are not recognized by mouse iNKT cells [50]. One recent study has identified peroxisome-derived, ether-bonded lipids as self antigens that can stimulate both mouse and human iNKT cells, and has suggested that these antigens are required for thymic iNKT cell development [53]. However, the potential role of these peroxisome-derived self antigens in the functional response of iNKT cells to microbes 
remains to be investigated. Another recent study has identified $\beta$-GluCer as a potent self antigen for both mouse and human iNKT cells [54]. $\beta$-GluCer accumulates during infection and in response to microbial products, and inhibition of its synthesis is able to inhibit iNKT cell activation by APCs exposed to microbial products. This study therefore suggests $\beta$ GluCer as a major self antigen for recognition of microbial products by iNKT cells. Whether $\beta$-GluCer is also induced during inflammatory conditions that are independent of microbial products is unknown. Regardless, it is unlikely that $\beta$-GluCer is the only self lipid that contributes to the response of iNKT cells to infection or inflammation. It is more likely that multipleand distinct self antigens contribute to iNKT cell activation during different inflammatory conditions.

\section{Additional mechanisms that may contribute to iNKT cell activation during inflammation}

A variety of additional mechanisms that can contribute to iNKT cell activation during inflammatory conditions have been identified (Figure 1c). (i) Expression of CD1d is regulated by the lipid-activated transcription factor peroxisome proliferator activated receptor (PPAR) $\gamma$ [55]. PPAR $\gamma$ ligands such as oxidized low-density lipoprotein (LDL) that are produced during inflammatory conditions might thus induce CD1d expression and promote iNKT cell autoreactivity [55,56]. (ii) Consistent with expression of the TCR V $\beta .8$ chain by a majority of murine iNKT cells, these cells have been shown to produce IFN- $\gamma$ in response to the staphylococcal enterotoxin $\mathrm{B}$, a superantigen, in a manner that requires interaction with MHC class II [57,58]. (iii) iNKT cells also constitutively express a low affinity $\mathrm{Fc} \gamma$ receptor ( $\mathrm{Fc} \gamma \mathrm{RIII}$ or CD16), and engagement of this receptor with antigenbound IgG antibodies results in IL-4 and IFN- $\gamma$ production, which contributes to disease induced in an experimental model of IgG-mediated joint inflammation [59]. (iv) iNKT cells can also receive signals via activating and inhibitory receptors on NK cells. Engagement of CD161 (NK1.1) on murine iNKT cells results in IFN- $\gamma$ production, even in the absence of TCR signaling [60], but ligation of this receptor on human iNKT cells only causes enhanced iNKT cell activation in the presence of TCR stimulation [61]. Similarly, ligation of the cellular stress ligand receptor NKG2D on human iNKT cells induced the lytic program in these cells [62]. (v) In addition to their response to NK cell receptor signals, iNKT cells can respond to a variety of activating and inhibitory $\mathrm{T}$ cell co-stimulatory molecules (reviewed in [63]). (vi) iNKT cells constitutively express the T cell immunoglobulin-like mucin-like (TIM)-1 molecule [64], which functions as a damage-associated molecular pattern for phosphatidylserine, a marker of apoptotic cells. TIM-1 recognition of apoptotic cells induces iNKT cell IL-4 and IFN- $\gamma$ secretion and this mechanism is suggested to contribute to the development of allergic airway hyper-reactivity in mice [64]. (vii) Although iNKT cells fail to express TLRs under noninflammatory conditions, a recent study has provided evidence that TCR engagement on iNKT cells can induce expression of several TLRs, and that subsequent stimulation with TLR agonists leads to enhanced iNKT cell activation [65]. (viii) A recent study has shown that hepatic iNKT cells can be directly activated to secrete IL-10 in response to neurotransmitters in an experimental model of stroke [66]. Thus, diverse mechanisms may contribute to iNKT cell activation during different inflammatory conditions.

\section{iNKT cells in inflammatory diseases}

iNKT cells have been implicated in immune defense against infection and cancer, and several autoimmune and inflammatory diseases. iNKT cells become activated in a variety of inflammatory conditions, resulting in numerical and functional alterations in these cells. The contribution of iNKT cells to disease can be either protective or pathogenic, depending on the particular disease investigated and the experimental model or mouse strain used. The immunomodulatory activities of iNKT cell antigens such as a-GalCer have been used extensively in preclinical studies to investigate their potential therapeutic applications, and 
based on the results obtained, several clinical trials have been conducted or are in progress (Box 2). Here, we review recent progress in understanding the contribution of iNKT cells to selected inflammatory diseases, and we refer to published reviews for earlier work [1-5,16]. A recurring theme in these studies is the variable and sometimes even conflicting results that have been obtained in different laboratories. Although this might reflect differences in the experimental models, mouse strains and methodologies used, another possibility is that these differences are due to variations in iNKT cell numbers and functions in mouse colonies from different institutions and vendors, due to differences in their endogenous microbiota (Box $3)$.

\section{Infection}

iNKT cells play a protective role in many infections, including those mediated by microorganisms that lack cognate iNKT cell antigens. iNKT cells also influence colonization of the gastrointestinal tract by commensal microbiota [67]. Some microorganisms such as Listeria monocytogenes upregulate CD1d expression, which might contribute to iNKT cell activation. By contrast, several viruses, including HIV-1, Kaposi sarcoma virus and herpes simplex virus 1 , downregulate CD1d expression, presumably as an immune evasion mechanism. iNKT cell activation is protective against infection by several microorganisms, but this is usually only effective within a narrow time window around the time of infection, thus limiting its therapeutic applications [16]. Although diverse mechanisms are likely at play, these protective effects of iNKT cells typically involve their capacity to produce IFN- $\gamma$ and to activate other cell types such as DCs, NK cells, and cytotoxic T cells, and, at least in the case of influenza virus infection, to overcome the suppressive effects of myeloid lineage cells [68]. Recent studies have further provided evidence that the protective effects of iNKT cells against lung damage during influenza virus infection involves production of IL-22 by these cells in response to activation of viral sensors in DCs [69]. Clinical trials with a-GalCer to treat hepatitis B or C virus infection have thus far been unsuccessful.

\section{Tumor immunity}

Studies in mice have provided evidence that iNKT cells contribute to natural tumor immunity against a variety of tumors, including chemically induced tumors, transplanted tumors, and tumors that arise in genetically altered mice. However, a recent study was unable to find evidence for a contribution of iNKT cells to methylcholanthrene-induced carcinomas [70]. Activation of iNKT cells with potent agonists leads to strong antimetastatic responses in mice, with the Th1-biasing agonists being most effective. Clinical trials with aGalCer, a-GalCer-pulsed DCs, in vitro expanded iNKT cells, and a-GalCer-pulsed DCs combined with in vitro expanded iNKT cells have been performed, with encouraging results $[71,72]$. Mechanisms involved in the anti-tumor activities of iNKT cells include the activation of downstream effectors such as NK cells, cytotoxic T cells, Th1 cells, and $\gamma \delta \mathrm{T}$ cells, production of IFN- $\gamma$, and direct lysis of suppressive myeloid lineage cells [16].

\section{Autoimmunity}

iNKT cells generally play a tolerogenic role in autoimmunity, including in experimental models of type 1 diabetes, multiple sclerosis, lupus, and arthritis. In many of these models, iNKT cell agonists protect against autoimmunity, but in some models, and depending on the mouse strain and treatment protocol used, iNKT cell activation exacerbates rather than protects against autoimmunity [16]. Disease protection is usually associated with enhanced Th2 and/or reduced Th1 responses against the target antigens involved. Nevertheless, although the Th2-biasing iNKT cell agonists are typically more effective than the Th1biasing agonists, in some models, Th1- and Th2-biasing reagents are equally effective. Therefore, mechanisms other than a shift in the Th1/Th2 balance of autoantigenspecific 
responses must be involved. Indeed, additional studies have provided evidence that iNKT cells can promote anergy in pathogenic $\mathrm{T}$ cells, and induce the generation of regulatory Foxp3-expressing T cells and suppressive myeloid-lineage cells [16].

\section{Allograft rejection}

iNKT cells can influence the immune response to allografts. iNKT cells have been shown to play a critical role in allograft models where tolerance is induced by blocking co-stimulatory receptors. iNKT cells are also essential for long-term survival of corneal allografts. However, iNKT cells can also contribute to allograft rejection, as observed in a model of pancreatic islet transplantation into the liver. Several studies have further provided evidence that iNKT cells can prevent graft versus host disease in an IL-4-dependent manner. A clinical trial investigating the capacity of a-GalCer to modulate allogeneic hematopoietic stem cell transplants is currently recruiting participants (http://clinicaltrials.gov/).

\section{Allergic disease and asthma}

iNKT cells play a critical role in allergic airway hyper-reactivity induced in mice by model allergens such as ovalbumin or ragweed, respiratory viruses, environmental pollutants such as ozone, house dust extracts, IL-25, iNKT cell antigens, and apoptotic respiratory epithelial cells [73]. However, iNKT cells can also play a protective role in lung inflammation, as shown in an experimental model of innate cell-driven lung inflammation induced by IL-33 [74], and in a model in which influenza virus infection of suckling mice protects against airway hyper-reactivity [21]. Treatment of allergic airway disease induced by ovalbumin or ragweed with a-GalCer either promotes or ameliorates disease, depending on the particular model used and the timing of a-GalCer administration [16]. The role of iNKT cells in the development of asthma in humans remains uncertain [73].

\section{Atherosclerosis}

CD1d molecules and iNKT cells are present in atherosclerotic lesions in mice and humans $[75,76]$. Several studies have shown that iNKT cells play a pathogenic role in the development of atherosclerosis in apolipoprotein (apo)E-deficient mice, LDL receptor (LDLR)-deficient mice, and mice fed an atherogenic diet [75]. Consistent with these findings, $\mathrm{a}$-GalCer treatment exacerbates atherogenesis in apoE-deficient mice. However, surprisingly, one study has reported that a-GalCer ameliorates atherosclerosis in LDLRdeficient mice [77].

\section{Colitis}

iNKT cells are critical for the development of Th2 type colitis induced by the hapten oxazolone in mice, with a critical role for IL-13, which is produced by iNKT cells and other innate lymphocytes in response to Th2-promoting cytokines such as IL-25 produced by intestinal epithelial cells [78]. Additional studies have shown that iNKT cell activation by aGalCer or $\beta$-GluCer is protective against Th1/Th17 type colitis induced by sodium dextran sulfate or trinitrobenzenesulfonic acid, respectively (reviewed in $[16,28]$ ).

\section{Metabolic disease}

As a result of the abundance of iNKT cells in the liver and adipose tissues, particularly human omentum [79], and because of their lipid-reactivity, several research groups have investigated the role of iNKT cells in the development of nonalcoholic fatty liver disease (NAFLD) and obesity-associated inflammation and insulin-resistance (reviewed in [80,81]). A decrease in liver NK1.1 $1^{+} \mathrm{T}$ cells has been observed in several mouse models of obesity [80]. However, the iNKT cell population appears to be expanded in human livers with NAFLD-related fibrosis. Conflicting results have been obtained for the role of iNKT cells in 
the development of NAFLD, with some studies providing evidence for a protective role, and others suggesting a pathogenic role of iNKT cells. However, in several of these studies no distinction was made between Type I and Type II NKT cells. Collectively, these studies have led to the hypothesis that hepatic iNKT cells play a protective role during the early phase of NAFLD but drive fibrogenesis during later stages of the disease process $[80,82]$. In models of obesity induced by a high-fat diet, iNKT cells in adipose tissue and liver rapidly adopt an activated phenotype $[83,84]$, and a similar phenotype is observed in adipose tissue of genetic obesity models [84]. In humans, iNKT cells are depleted in the omentum of obese individuals [79]. One study has reported that adoptive transfer of $\mathrm{NK} 1.1^{+} \mathrm{CD} 3^{+} \mathrm{T}$ cells ameliorates fatty liver and glucose intolerance in leptin-deficient ob/ob mice [85], and another study has reported that adoptive transfer of NK1.1 $1^{+} \mathrm{TCR}^{+}$cells from mice fed a normal chow diet ameliorates insulin resistance in mice on a high-fat diet [86], suggesting a protective role of NKT cells in obesity-induced metabolic disease. However, comparing obese wild-type, CD1d-deficient and/or Ja18-deficient mice, some studies have been unable to obtain evidence for a role of iNKT cells [87-90], whereas other studies have found evidence for either an ameliorating [83] or pathogenic role [84,91] of iNKT cells in obesityassociated inflammation, fatty liver disease, and insulin resistance. Finally, another study, using wild-type, CD1d-deficient and Ja18-deficient mice on a low-fat diet, has provided evidence for a protective role of NKT cells in lean adipose tissue against insulin resistance [90]. Adding to these divergent findings, iNKT cell antigens have been reported either to ameliorate [92,93], not affect [90,91], or exacerbate [84,87,91] metabolic disease. These divergent findings might be caused by a variety of experimental parameters that differed between these studies; most notably differences in the endogenous microbiota that are present in the animal facilities where the mice were housed (Box 3). In this respect, it is interesting that one study has suggested that NKT cells contribute to the capacity of probiotics to improve high-fat-diet-induced metabolic disease [86].

\section{Ischemia-reperfusion injury}

iNKT cells expand and become activated during ischemia-reperfusion injury in several organs. These cells have been shown to play a critical role in ischemia-reperfusion injury induced in liver and kidney. iNKT cells are also important for the development of ischemiareperfusion injury in a mouse model of sickle cell disease [94]. Interestingly, a recent study has shown that hepatic iNKT cells play a critical role in the induction of immune suppression after stroke, in a mechanism that involves iNKT cell stimulation by a noradrenergic neurotransmitter [66].

\section{Inflammation-induced preterm delivery}

CD1d molecules and iNKT cells are present at the fetal-maternal interface. Activation of these cells with a-GalCer during pregnancy can cause abortion. A more recent study has provided evidence that iNKT cells are essential for the induction of preterm birth mediated by LPS [95], suggesting that these cells may play an essential role in inflammation-induced preterm birth.

\section{Concluding remarks}

iNKT cells are early sensors of inflammation. The strategic intravascular location of these cells in several organs permits their rapid response to blood-borne or environmental insults. Recent studies have provided important insight into the mechanisms that permit iNKT cells to become activated in a variety of inflammatory conditions. The effector functions of iNKT cells are modulated by several factors such as environmental microbiota, which might have an important impact on the contributions of these cells to disease. The preclinical studies with a-GalCer and other iNKT cell antigens have raised substantial enthusiasm for 
developing iNKT cell-based adjuvants and immunotherapies. Although iNKT cell activation in humans appears to be safe, inducing the biological responses of these cells in humans has been challenging. Nevertheless, encouraging results have been obtained in some clinical trials with cancer patients. Future studies in this area (outstanding questions, Box 4) should be instrumental for harnessing the immunomodulatory properties of iNKT cells to treat human inflammatory diseases.

\section{Acknowledgments}

We apologize to investigators whose work we did not cite due to space constraints or omission. We thank Dr Sebastian Joyce for helpful discussions and support. Work in the authors' laboratory was supported by grants from the National Institutes of Health. V.V.P. was supported by a postdoctoral fellowship from the National Multiple Sclerosis Society.

\section{References}

1. Taniguchi M, et al. The regulatory role of Va14 NKT cells in innate and acquired immune response. Annu. Rev. Immunol. 2003; 21:483-513. [PubMed: 12543936]

2. Brigl M, Brenner MB. CD1: antigen presentation and T cell function. Annu. Rev. Immunol. 2004; 22:817-890. [PubMed: 15032598]

3. Kronenberg M. Toward an understanding of NKT cell biology: progress and paradoxes. Annu. Rev. Immunol. 2005; 26:877-900. [PubMed: 15771592]

4. Bendelac A, et al. The biology of NKT cells. Annu. Rev. Immunol. 2007; 25:297-336. [PubMed: 17150027]

5. Van Kaer L. NKT cells: T lymphocytes with innate effector functions. Curr. Opin. Immunol. 2007; 19:354-364. [PubMed: 17428648]

6. Liu Y, et al. Endogenous collagen peptide activation of CD1d-restricted NKT cells ameliorates tissue-specific inflammation in mice. J. Clin. Invest. 2011; 121:249-264. [PubMed: 21157037]

7. Geissmann $\mathrm{F}$, et al. Intravascular immune surveillance by $\mathrm{CXCR}^{+} \mathrm{NKT}$ cells patrolling liver sinusoids. PLoS Biol. 2005; 3:650-661.

8. Scanlon ST, et al. Airborne lipid antigens mobilize resident intravascular NKT cells to induce allergic airway inflammation. J. Exp. Med. 2011; 208:2113-2124. [PubMed: 21930768]

9. Michel ML, et al. Identification of an IL-17-producing NK1.1- ${ }^{-}$iNKT cell population involved in airway neutrophilia. J. Exp. Med. 2007; 204:995-1001. [PubMed: 17470641]

10. Doisne JM, et al. Cutting edge: crucial role of IL-1 and IL-23 in the innate IL-17 response of peripheral lymph node NK1.1- ${ }^{-}$invariant NKT cells to bacteria. J. Immunol. 2011; 186:662-666. [PubMed: 21169541]

11. Chang PP, et al. Identification of Bcl-6-dependent follicular helper NKT cells that provide cognate help for B cell responses. Nat. Immunol. 2012; 13:35-43. [PubMed: 22120117]

12. King IL, et al. Invariant natural killer $\mathrm{T}$ cells direct $\mathrm{B}$ cell responses to cognate lipid antigen in an IL-21-dependent manner. Nat. Immunol. 2012; 13:44-50. [PubMed: 22120118]

13. Tonti E, et al. Follicular helper NKT cells induce limited B cell responses and germinal center formation in the absence of CD4 ${ }^{+} \mathrm{T}$ cell help. J. Immunol. 2012; 188:3217-3222. [PubMed: 22379027]

14. Monteiro M, et al. Identification of regulatory Foxp $3^{+}$invariant NKT cells induced by TGF-b. J. Immunol. 2010; 185:2157-2163. [PubMed: 20639482]

15. Moreira-Teixeira L, et al. Rapamycin combined with TGF-b converts human invariant NKT cells into suppressive Foxp3 ${ }^{+}$regulatory cells. J. Immunol. 2012; 188:624-631. [PubMed: 22156591]

16. Van Kaer L, et al. Invariant NK T cells: potential for immunotherapeutic targeting with glycolipid antigens. Immunotherapy. 2011; 3:59-75. [PubMed: 21174558]

17. Kovalovsky D, et al. The BTB-zinc finger transcriptional regulator PLZF controls the development of invariant natural killer T cell effector functions. Nat. Immunol. 2008; 9:1055-1064. [PubMed: 18660811] 
18. Savage AK, et al. The transcription factor PLZF directs the effector program of the NKT cell lineage. Immunity. 2008; 29:391-403. [PubMed: 18703361]

19. Brigl M, Brenner MB. How invariant natural killer T cells respond to infection by recognizing microbial or endogenous lipid antigens. Semin. Immunol. 2010; 22:79-86. [PubMed: 19948416]

20. Kinjo Y, et al. Invariant natural killer T cells recognize glycolipids from pathogenic Gram-positive bacteria. Nat. Immunol. 2011; 12:966-974. [PubMed: 21892173]

21. Chang YJ, et al. Influenza infection in suckling mice expands an NKT cell subset that protects against airway hyperreactivity. J. Clin. Invest. 2011; 121:57-69. [PubMed: 21157038]

22. Agea E, et al. Human CD1-restricted T cell recognition of lipids from pollens. J. Exp. Med. 2005; 202:295-308. [PubMed: 16009719]

23. Wingender G, et al. Invariant NKT cells are required for airway inflammation induced by environmental antigens. J. Exp. Med. 2011; 208:1151-1162. [PubMed: 21624935]

24. Kawano T, et al. CD1d-restricted and TCR-mediated activation of Va14 NKT cells by glycosylceramides. Science. 1997; 278:1626-1629. [PubMed: 9374463]

25. Mallevaey T, Selvanantham T. Strategy of lipid recognition by invariant natural killer T cells: 'one for all and all for one'. Immunology. 2012; 136:273-282. [PubMed: 22671023]

26. Parekh VV, et al. Quantitative and qualitative differences in the in vivo response of NKT cells to distinct a- and b-anomeric glycolipids. J. Immunol. 2004; 173:3693-3706. [PubMed: 15356115]

27. Ortaldo JR, et al. Dissociation of NKT stimulation, cytokine induction, and NK activation in vivo by the use of distinct TCR-binding ceramides. J. Immunol. 2004; 172:943-953. [PubMed: 14707067]

28. Ilan Y. Alpha versus beta: are we on the way to resolve the mystery as to which is the endogenous ligand for natural killer T cells? Clin. Exp. Immunol. 2009; 158:300-307. [PubMed: 19793337]

29. Brigl M, et al. Innate and cytokine-driven signals, rather than microbial antigens, dominate in natural killer T cell activation during microbial infection. J. Exp. Med. 2011; 208:1163-1177. [PubMed: 21555485]

30. Brigl M, et al. Mechanism of CD1d-restricted natural killer T cell activation during microbial infection. Nat. Immunol. 2003; 4:1230-1237. [PubMed: 14578883]

31. Tyznik AJ, et al. Cutting edge: the mechanism of invariant NKT cell responses to viral danger signals. J. Immunol. 2008; 181:4452-4456. [PubMed: 18802047]

32. Wesley JD, et al. NK cell-like behavior of Va14i NK T cells during MCMV infection. PLoS Pathog. 2008; 4:e1000106. [PubMed: 18636102]

33. Paget C, et al. Activation of invariant NKT cells by toll-like receptor 9-stimulated dendritic cells requires type I interferon and charged glycosphingolipids. Immunity. 2007; 27:597-609. [PubMed: 17950005]

34. Salio M, et al. Modulation of human natural killer T cell ligands on TLR-mediated antigenpresenting cell activation. Proc. Natl. Acad. Sci. U.S.A. 2007; 104:20490-20495. [PubMed: 18077358]

35. Cohen NR, et al. Innate recognition of cell wall b-glucans drives invariant natural killer T cell responses against fungi. Cell Host Microbe. 2011; 10:437-450. [PubMed: 22100160]

36. Nagarajan NA, Kronenberg M. Invariant NKT cells amplify the innate immune response to lipopolysaccharide. J. Immunol. 2007; 178:2706-2713. [PubMed: 17312112]

37. Raghuraman G, et al. IFN-b-mediated up-regulation of CD1d in bacteria-infected APCs. J. Immunol. 2006; 177:7841-7848. [PubMed: 17114455]

38. Khovidhunkit W, et al. Effects of infection and inflammation on lipid and lipoprotein metabolism: mechanisms and consequences to the host. J. Lipid Res. 2004; 45:1169-1196. [PubMed: $15102878]$

39. Muindi K, et al. Activation state and intracellular trafficking contribute to the repertoire of endogenous glycosphingolipids presented by CD1d. Proc. Natl. Acad. Sci. U.S.A. 2010; 107:3052-3057. [PubMed: 20133624]

40. Mattner J, et al. Exogenous and endogenous glycolipid antigens activate NKT cells during microbial infections. Nature. 2005; 434:525-529. [PubMed: 15791258] 
41. Darmoise A, et al. Lysosomal a-galactosidase controls the generation of self lipid antigens for natural killer T cells. Immunity. 2010; 33:216-228. [PubMed: 20727792]

42. Stanic AK, et al. Defective presentation of the CD1d1-restricted natural Va14Ja18 NKT lymphocyte antigen caused by b-D-glucosylceramide synthase deficiency. Proc. Natl. Acad. Sci. U.S.A. 2003; 100:1849-1854. [PubMed: 12576547]

43. Chiu YH, et al. Multiple defects in antigen presentation and T cell development by mice expressing cytoplasmic tail-truncated CD1d. Nat. Immunol. 2002; 3:55-60. [PubMed: 11731798]

44. Zhou D, et al. Lysosomal glycosphingolipid recognition by NKT cells. Science. 2004; 306:17861789. [PubMed: 15539565]

45. Speak AO, et al. Implications for invariant natural killer $\mathrm{T}$ cell ligands due to the restricted presence of isoglobotrihexosylceramide in mammals. Proc. Natl. Acad. Sci. U.S.A. 2007; 104:5971-5976. [PubMed: 17372214]

46. Li Y, et al. Immunologic glycosphingolipidomics and NKT cell development in mouse thymus. J. Proteome Res. 2009; 8:2740-2751. [PubMed: 19284783]

47. Porubsky S, et al. Normal development and function of invariant natural killer T cells in mice with isoglobotrihexosylceramide (iGb3) deficiency. Proc. Natl. Acad. Sci. U.S.A. 2007; 104:59775982. [PubMed: 17372206]

48. Chen X, et al. Distinct endosomal trafficking requirements for presentation of autoantigens and exogenous lipids by human CD1d molecules. J. Immunol. 2007; 178:6181-6190. [PubMed: 17475845]

49. Porubsky S, et al. Globosides but not isoglobosides can impact the development of invariant NKT cells and their interaction with dendritic cells. J. Immunol. 2012; 189:3007-3017. [PubMed: 22875802]

50. Pei B, et al. Diverse endogenous antigens for mouse NKT cells: self-antigens that are not glycosphingolipids. J. Immunol. 2011; 186:1348-1360. [PubMed: 21191069]

51. Gumperz JE, et al. Murine CD1d-restricted T cell recognition of cellular lipids. Immunity. 2000; 12:211-221. [PubMed: 10714687]

52. Fox LM, et al. Recognition of lyso-phospholipids by human natural killer T lymphocytes. PLoS Biol. 2009; 7:e1000228. [PubMed: 19859526]

53. Facciotti F, et al. Peroxisome-derived lipids are self antigens that stimulate invariant natural killer T cells in the thymus. Nat. Immunol. 2012; 13:474-480. [PubMed: 22426352]

54. Brennan PJ, et al. Invariant natural killer $\mathrm{T}$ cells recognize lipid self antigen induced by microbial danger signals. Nat. Immunol. 2011; 12:1202-1211. [PubMed: 22037601]

55. Szatmari I, et al. Activation of PPARg specifies a dendritic cell subtype capable of enhanced induction of iNKT cell expansion. Immunity. 2004; 21:95-106. [PubMed: 15345223]

56. Nakai Y, et al. Natural killer T cells accelerate atherogenesis in mice. Blood. 2004; 104:20512059. [PubMed: 15113755]

57. Rieder SA, et al. CD1d-independent activation of invariant natural killer T cells by staphylococcal enterotoxin B through major histocompatibility complex class II/T cell receptor interaction results in acute lung injury. Infect. Immun. 2011; 79:3141-3148. [PubMed: 21628519]

58. Hayworth JL, et al. CD1d-independent activation of mouse and human iNKT cells by bacterial superantigens. Immunol. Cell Biol. 2012; 90:699-709. [PubMed: 22041925]

59. Kim HY, et al. FcgRIII engagement provides activating signals to NKT cells in antibody-induced joint inflammation. J. Clin. Invest. 2006; 116:2484-2492. [PubMed: 16917543]

60. Arase $\mathrm{H}$, et al. Interferon g production by natural killer $(\mathrm{NK})$ cells and NK1.1 ${ }^{+} \mathrm{T}$ cells upon NKRP1 cross-linking. J. Exp. Med. 1996; 183:2391-2396. [PubMed: 8642351]

61. Exley M, et al. CD161 (NKR-P1A) costimulation of CD1d-dependent activation of human T cells expressing invariant Va24 JaQ T cell receptor a chains. J. Exp. Med. 1998; 188:867-876. [PubMed: 9730888]

62. Kuylenstierna C, et al. NKG2D performs two functions in invariant NKT cells: direct TCRindependent activation of NK-like cytolysis and co-stimulation of activation by CD1d. Eur. J. Immunol. 2011; 41:1913-1923. [PubMed: 21590763] 
63. van den Heuvel MJ, et al. NKT cell costimulation: experimental progress and therapeutic promise. Trends Mol. Med. 2011; 17:65-77. [PubMed: 21087900]

64. Lee HH, et al. Apoptotic cells activate NKT cells through T cell Ig-like mucin-like-1 resulting in airway hyperreactivity. J. Immunol. 2010; 185:5225-5235. [PubMed: 20889552]

65. Kulkarni RR, et al. Costimulatory activation of murine invariant natural killer T cells by toll-like receptor agonists. Cell. Immunol. 2012; 277:33-43. [PubMed: 22795895]

66. Wong $\mathrm{CH}$, et al. Functional innervation of hepatic iNKT cells is immunosuppressive following stroke. Science. 2011; 334:101-105. [PubMed: 21921158]

67. Nieuwenhuis EE, et al. Cd1d-dependent regulation of bacterial colonization in the intestine of mice. J. Clin. Invest. 2009; 119:1241-1250. [PubMed: 19349688]

68. De Santo C, et al. Invariant NKT cells reduce the immunosuppressive activity of influenza A virusinduced myeloid-derived suppressor cells in mice and humans. J. Clin. Invest. 2008; 118:40364048. [PubMed: 19033672]

69. Paget $\mathrm{C}$, et al. Interleukin-22 is produced by invariant natural killer $\mathrm{T}$ lymphocytes during influenza A virus infection: potential role in protection against lung epithelial damages. J. Biol. Chem. 2012; 287:8816-8829. [PubMed: 22294696]

70. Kammertoens T, et al. B-cells and IL-4 promote methylcholanthrene-induced carcinogenesis but there is no evidence for a role of T/NKT-cells and their effector molecules (Fas-ligand, TNF-a, perforin). Int. J. Cancer. 2012; 131:1499-1508. [PubMed: 22212899]

71. Motohashi S, et al. A phase I-II study of a-galactosylceramide-pulsed IL-2/GM-CSF-cultured peripheral blood mononuclear cells in patients with advanced and recurrent non-small cell lung cancer. J. Immunol. 2009; 182:2492-2501. [PubMed: 19201905]

72. Kunii N, et al. Combination therapy of in vitro-expanded natural killer T cells and agalactosylceramide-pulsed antigen-presenting cells in patients with recurrent head and neck carcinoma. Cancer Sci. 2009; 100:1092-1098. [PubMed: 19302288]

73. Iwamura C, Nakayama T. Role of NKT cells in allergic asthma. Curr. Opin. Immunol. 2010; 22:807-813. [PubMed: 21115332]

74. Bourgeois EA, et al. A natural protective function of invariant NKT cells in a mouse model of innate-cell-driven lung inflammation. Eur. J. Immunol. 2011; 41:299-305. [PubMed: 21268000]

75. Braun NA, et al. Natural killer T cells and atherosclerosis: form and function meet pathogenesis. J. Innate Immun. 2010; 2:316-324. [PubMed: 20375560]

76. Kyriakakis E, et al. Invariant natural killer T cells: linking inflammation and neovascularization in human atherosclerosis. Eur. J. Immunol. 2010; 40:3268-3279. [PubMed: 21061446]

77. van Puijvelde GH, et al. Effect of natural killer T cell activation on the initiation of atherosclerosis. Thromb. Haemost. 2009; 102:223-230. [PubMed: 19652872]

78. Camelo, A., et al. Blocking IL-25 signalling protects against gut inflammation in a type-2 model of colitis by suppressing nuocyte and NKT derived IL-13.. J. Gastroenterol. 2012. http://dx.doi.org/ $10.1007 / \mathrm{s} 00535-012-0591-2$

79. Lynch L, et al. Invariant NKT cells and CD1d ${ }^{+}$cells amass in human omentum and are depleted in patients with cancer and obesity. Eur. J. Immunol. 2009; 39:1893-1901. [PubMed: 19585513]

80. Tajiri, K.; Shimizu, Y. Role of NKT cells in the pathogenesis of NAFLD.. Int. J. Hepatol. 2012. http://dx.doi.org/10.1155/2012/850836

81. Wu L, Van Kaer L. Contribution of lipid-reactive natural killer T cells to obesity-associated inflammation and insulin resistance. Adipocyte. 2012 (in press).

82. Syn WK, et al. NKT-associated hedgehog and osteopontin drive fibrogenesis in non-alcoholic fatty liver disease. Gut. 2012; 61:1323-1329. [PubMed: 22427237]

83. Ji Y, et al. Short-term high-fat-diet challenge promotes alternative macrophage polarization in adipose tissue via natural killer T cells and interleukin-4. J. Biol. Chem. 2012; 287:24378-24386. [PubMed: 22645141]

84. Wu L, et al. Activation of invariant natural killer T cells by lipid excess promotes tissue inflammation, insulin resistance, and hepatic steatosis in obese mice. Proc. Natl. Acad. Sci. U.S.A. 2012; 109:E1143-E1152. [PubMed: 22493234] 
85. Elinav E, et al. Adoptive transfer of regulatory NKT lymphocytes ameliorates non-alcoholic steatohepatitis and glucose intolerance in ob/ob mice and is associated with intrahepatic CD8 trapping. J. Pathol. 2006; 209:121-128. [PubMed: 16482497]

86. Ma X, et al. Probiotics improve high fat diet-induced hepatic steatosis and insulin resistance by increasing hepatic NKT cells. J. Hepatol. 2008; 49:821-830. [PubMed: 18674841]

87. Satoh M, et al. Type II NKT cells stimulate diet-induced obesity by mediating adipose tissue inflammation, steatohepatitis and insulin resistance. PLoS ONE. 2012; 7:e30568. [PubMed: 22383967]

88. Kotas ME, et al. Impact of CD1d deficiency on metabolism. PLoS ONE. 2011; 6:e25478. [PubMed: 21980475]

89. Mantell BS, et al. Mice lacking NKT cells but with a complete complement of CD8 ${ }^{+}$T-cells are not protected against the metabolic abnormalities of diet-induced obesity. PLoS ONE. 2011; 6:e19831. [PubMed: 21674035]

90. Schipper HS, et al. Natural killer T cells in adipose tissue prevent insulin resistance. J. Clin. Invest. 2012; 122:3343-3354. [PubMed: 22863618]

91. Ohmura K, et al. Natural killer T cells are involved in adipose tissues inflammation and glucose intolerance in diet-induced obese mice. Arterioscler. Thromb. Vasc. Biol. 2010; 30:193-199. [PubMed: 19910631]

92. Ji Y, et al. Activation of natural killer T cells promotes M2 macrophage polarization in adipose tissue and improves systemic glucose tolerance via interleukin-4 (IL-4)/STAT6 protein signaling axis in obesity. J. Biol. Chem. 2012; 287:13561-13571. [PubMed: 22396530]

93. Margalit M, et al. Glucocerebroside ameliorates the metabolic syndrome in OB/OB mice. J. Pharmacol. Exp. Ther. 2006; 319:105-110. [PubMed: 16815870]

94. Field JJ, et al. Targeting iNKT cells for the treatment of sickle cell disease. Clin. Immunol. 2011; 140:177-183. [PubMed: 21429807]

95. Li LP, et al. Depletion of invariant NKT cells reduces inflammation-induced preterm delivery in mice. J. Immunol. 2012; 188:4681-4689. [PubMed: 22467647]

96. Godfrey DI, et al. NKT cells: what's in a name? Nat. Rev. Immunol. 2004; 4:231-237. [PubMed: 15039760]

97. Uldrich AP, et al. A semi-invariant $\mathrm{Va}_{10}{ }^{+} \mathrm{T}$ cell antigen receptor defines a population of natural killer T cells with distinct glycolipid antigen-recognition properties. Nat. Immunol. 2011; 12:616623. [PubMed: 21666690]

98. Bedel R, et al. Lower TCR repertoire diversity in Traj18-deficient mice. Nat. Immunol. 2012; 13:705-706. [PubMed: 22814339]

99. Arrenberg P, et al. Oligoclonality and innate-like features in the TCR repertoire of type II NKT cells reactive to a b-linked self-glycolipid. Proc. Natl. Acad. Sci. U.S.A. 2010; 107:10984-10989. [PubMed: 20534460]

100. Jahng A, et al. Prevention of autoimmunity by targeting a distinct, noninvariant CD1d-reactive T cell population reactive to sulfatide. J. Exp. Med. 2004; 199:947-957. [PubMed: 15051763]

101. Rhost, S., et al. Identification of novel glycolipid ligands activating a sulfatide reactive, CD1drestricted, type II natural killer T lymphocyte.. Eur. J. Immunol. 2012. http://dx.doi.org/10.1002/ eji.201142350 (in press)

102. Blumberg R, Powrie F. Microbiota, disease, and back to health: a metastable journey. Sci. Transl. Med. 2012; 4:137rv137.

103. Wei B, et al. Commensal microbiota and $\mathrm{CD} 8^{+} \mathrm{T}$ cells shape the formation of invariant NKT cells. J. Immunol. 2010; 184:1218-1226. [PubMed: 20048124]

104. Olszak T, et al. Microbial exposure during early life has persistent effects on natural killer T cell function. Science. 2012; 336:489-493. [PubMed: 22442383]

105. Wingender $\mathrm{G}$, et al. Intestinal microbes affect phenotypes and functions of invariant natural killer T cells in mice. Gastroenterology. 2012; 143:418-428. [PubMed: 22522092] 


\section{Box 1. NKT cell subsets and related cell types}

NKT cells are defined as lymphocytes that coexpress a TCR with markers that are typical of the NK cell lineage, and depend on the CD1d molecule for their development and function [96]. Two main subsets of NKT cells have been identified. Type I NKT cells, which are usually referred to as iNKT cells, express a semi-invariant TCR, consisting of Va14-Ja 18 paired with V $\beta 8.2$, 7, or 2 in mice or the homologous Va24-Ja 18 paired with V $\beta 11$ in humans. These cells react with the marine sponge-derived glycosphingolipid $a-G a l C e r$, and therefore can be identified most reliably with multimeric CD1d molecules loaded with synthetic a-GalCers (such as KRN7000 or PBS-57). These cells are absent in both CD1d- and Ja18-deficient mice. However, even this definition of iNKT cells is imprecise, because a small subset of a-GalCer-reactive NKT cells expressing a canonical Va10-Ja50 TCRa chain was recently identified in both mice and humans [97]. These cells showed a preference for reactivity with aGluCer and a-glucuronic-acid-containing glycolipids, and were absent in CD1d-deficient mice but present in Ja 18-deficient mice. Furthermore, a recent study has provided evidence that Ja18-deficient mice have unexpected alterations in their TCR repertoire that go beyond the absence of iNKT cells, cautioning against the extensive use of these animals to dissect the relative role of distinct NKT cell populations in immune responses and disease [98]. Type II NKT cells, also referred to as non-iNKT cells or variant NKT (vNKT) cells, express more diverse TCRs than Type I NKT cells, although the TCR repertoire of these cells contains a few oligoclonal populations [99]. A subset of type II NKT cells reacts with $\beta$-linked glycolipids such as the self-antigens sulfatide, $\beta$-GluCer and $\beta$-GalCer $[54,100,101]$. Type II NKT cells are usually identified as

NK1. $1^{+} \mathrm{TCR}^{+}{ }^{+} \mathrm{CD} 1 \mathrm{~d}^{-}$tetramer ${ }^{-}$cells, but this is imprecise. These cells are absent from CD1d-deficient mice but are present in Ja18-deficient mice. Type II NKT cells have been implicated in the development of autoimmunity, tumor immunity, metabolic disease, and viral hepatitis in mice. Although Type I NKT cells are the predominant NKT cell subset in mice, Type II NKT cells appear to be more abundant than Type I NKT cells in humans. In addition to these CD1d-dependent $\mathrm{T}$ cell populations, additional $\mathrm{T}$ cell subsets that express NK cell markers under certain conditions have been identified [96]. Some of these cells are selected by MHC-class-I-related proteins such as MHC-class-Irelated 1 (MR1), or might represent classical T cells that induced NK1.1 expression upon activation. These cells do not require CD1d expression for their development or function, therefore, they are present in CD1d-deficient animals. 
Box 2. Immunomodulatory, adjuvant, and therapeutic activities of iNKT cell antigens

The prototypic iNKT cell antigen a-GalCer, which is recognized at very high affinity by the TCR of iNKT cells, was originally isolated from a marine sponge during a screen for products with antimeta-static activities in mice [24]. This discovery subsequently led many investigators to explore the immunomodulatory and adjuvant activities of synthetic versions of a-GalCer and numerous structural analogs [1-5]. Collectively, these studies have shown that activation of iNKT cells results in the modulation of a variety of other cell types, including myeloid cells, NK cells, and cells of the adaptive immune system. As a result of their potent capacity to activate DCs, iNKT cell antigens hold great promise as vaccine adjuvants. The therapeutic activities of iNKT cell antigens have been extended to a variety of diseases, including infections, cancer metastases, auto-immunity, and some inflammatory diseases. However, iNKT cell activation can also promote inflammatory responses, such as allergic airway inflammation and atherosclerosis. Glycolipid antigens that preferentially induce the proinflammatory and adjuvant activities of iNKT cells (typically associated with a Th1 cytokine production profile by iNKT cells) or the anti-inflammatory and tolerogenic activities of these cells (typically associated with a Th2 cytokine production profile by iNKT cells) have been identified [16]. Multiple mechanisms have been suggested to contribute to the cytokine bias of iNKT cells, including the strength of the interaction with the invariant $\mathrm{T}$ cell receptor, the location where the antigens are loaded onto CD1d molecules, presentation by CD1d molecules localized in lipid rafts, in vivo pharmacological properties, and display by distinct APCs. Based on these preclinical studies in mice, several clinical trials exploring the therapeutic activities of iNKT cell antigens against cancer and hepatitis virus infections have been performed, and additional trials are underway (http://clinicaltrials.gov/). Some encouraging results for clinical trials with cancer patients have been obtained [71,72]. 


\section{Box 3. iNKT cell effector functions and commensal microbiota}

Commensal microbes play an important role in shaping the immune system and can influence the development of a variety of diseases, including inflammatory bowel disease, asthma, metabolic disease, and cancer [102]. Recent studies have shown that microbiota, some of which carry iNKT cell antigens, can influence the numbers, phenotypes and functions of iNKT cells in mice [103-105]. iNKT cells from the spleen and liver of germ-free mice, as compared with iNKT cells from mice exposed to a normal microbial environment, are reduced in number, have a less mature phenotype, and are hyporesponsive to activation with a-GalCer. iNKT cells from germ-free mice express the chemokine ligand CXCL16, causing these cells to accumulate in the colonic lamina propria and lung, which results in increased disease in models of inflammatory bowel disease and asthma that are controlled by iNKT cells [104]. Importantly, colonization of germ-free mice with conventional microbiota prevents this iNKT accumulation in mucosa and reduces disease susceptibility. However, accumulation of iNKT cells in the mucosa of germ-free mice is reversible in neonatal but not adult mice, suggesting that microbial exposure during early life has persistent effects on the iNKT cell population. These findings provide strong evidence that microbiota can have long-lasting effects on iNKT cell functions. These studies also imply that animals purchased from different vendors or housed in different animal facilities, and thus exposed to distinct groups of environmental microbiota, exhibit variations in iNKT cell functions. This could potentially explain some of the divergent results that have been obtained in different laboratories for the contribution of iNKT cells to certain diseases. Furthermore, differences in microbial colonization might also contribute to the wide differences in the abundance of iNKT cells in the human population. 


\section{Box 4. Outstanding questions}

- What are the relevant stimuli that contribute to iNKT cell activation during diverse inflammatory conditions?

- When iNKT cell activation involves the autoreactivity of these cells, do self antigens such as $\beta$-GluCer, iGb3, lysosulfatide or peroxisome-derived lipids play a role? How many self lipids are involved? What are the mechanisms that control the induction of these self antigens?

- How similar are the mechanisms that control iNKT cell activation in response to inflammation in mice and humans? How do they differ?

- How can an improved understanding of iNKT cell activation during inflammation be exploited to suppress or facilitate iNKT cell responses for immunotherapy?

- How do environmental microbiota influence the contribution of iNKT cells to disease processes that are controlled by these cells? How do microbiota influence the therapeutic properties of these cells?

- How do alterations in iNKT cell numbers and functions that have been observed in a variety of inflammatory diseases impact the therapeutic capacity of iNKT cells? Are these alterations reversible? 


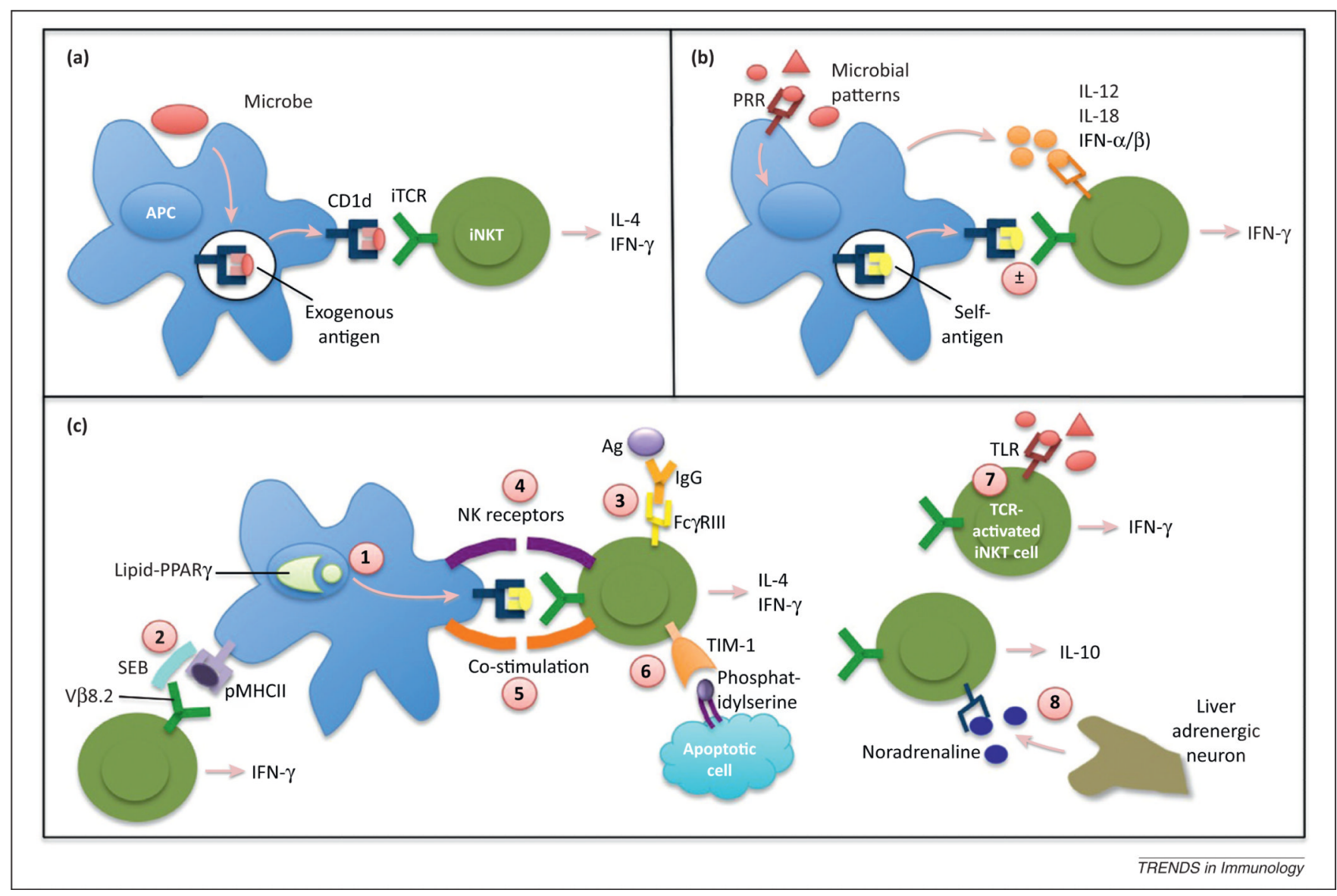

Figure 1.

Mechanisms of invariant natural killer T (iNKT) cell activation during inflammation. (a) Direct activation of iNKT cells by interaction of the invariant T cell receptor (iTCR) with exogenous antigens presented by CD1d molecules on antigen-presenting cells (APCs). (b) Indirect activation of iNKT cells by cytokines such as interleukin (IL)-12 produced by APCs in response to agonists of pattern-recognition receptors (PRRs) in the presence (+) or absence (-) of weak interactions of the iTCR with self antigens. (c) Additional mechanisms that can contribute to iNKT cell activation during inflammation in certain situations: (i) signaling of the peroxisome proliferator activated receptor (PPAR) $\gamma$ in response to lipid agonists might induce CD1d expression; (ii) superantigens such as staphylococcal enterotoxin B (SEB) might crosslink peptide-major histocompatibility complex (MHC) class II (pMHCII) complexes and certain TCR V $\beta$ chains (V $\beta 8.2$ in the case of SEB) on iNKT cells; (iii) antigen (Ag)-IgG complexes might activate Fc $\gamma$ RIII on iNKT cells; (iv) NK cell receptors on iNKT cells might be engaged by their counter-receptors on APCs; (v) co-stimulatory receptors on iNKT cells might be engaged by their counter-receptors on APCs; (vi) T cell immunoglobulin-like mucin-like (TIM)-1 on iNKT cells might bind with phosphatidylserine on apoptotic cells; (vii) TCR-activated iNKT cells might induce expression of Toll-like receptors (TLRs) that can be engaged by their ligands; and (viii) neurotransmitters such as noradrenaline might interact with their receptors on iNKT cells. iNKT cells activated by these diverse signals may produce a variety of cytokines, as indicated. 